23) Toyama, A.: M. S. Thesis of Rice Univ. (1966)

24) Wichterle, I. and R. Kobayashi: J. Chem. Eng. Data, 17, 4 (1972)

25) Wichterle, I. and R. Kobayashi: J. Chem. Eng. Data, 17, 9
(1972)

26) Yen, L. C. S. and J. J. Mcketta: AIChE J., 8, 501 (1962)

27) Zellner, M. G., L. C. Claitor and J. M. Prausnitz: Ind. Eng. Chem., Fundamentals, 9, 549 (1970)

\title{
PHENOMENAL TRANSITION OF LIQUID ATOMIZATION FROM DISK*
}

\author{
Shiro MATSUMOTO, Kuniki SAITO AND Yoichi TAKASHIMA \\ Research Laboratory of Nuclear Reactor, Tokyo Institute of Technology, Tokyo
}

\begin{abstract}
The change of atomization mechanism at the edge of a rotating disk was studied based on theoretical consideration and various experimental data.

The flow rate at the transition was determined by seeing directly the transition phenomena with a stroboscope. Four kinds of liquids and five kinds of disks were used in this experiment, and the rotational number was varied from 500 to $4000 \mathrm{rpm}$.

As a result, it was seen that the transition accompanied a kind of hysteresis phenomenon. The flow rate at the transition from direct drop to ligament formation was a little more than that at the reverse transition, as given by Eqs.(27) and (28), respectively. Also, for the transition from ligament to film formation and the reverse transition, a similar hysteresis was obtained. Hinze and Milborn's equation can be used for liquid of less than a few poises in viscosity for prediction of the flow rate at the transition from ligament to film formation, and the equation by Tanasawa $e t$ al. is applicable to more viscous liquids.
\end{abstract}

\section{Introduction}

Spinning-disk atomizers are widely used for gasliquid contact processes such as spray drying, cooling, scrubbing and combustion. It is known that three different types of drop formation may take place around and beyond the edge of a rotating disk.

Figure 1 illustrates these three different types of disintegration. At a very low flow rate, direct drop formation occurs around the edge, as shown in Fig. 1-a. As the flow rate increases, the size of drops removed from the disk remains constant, and the frequency of drop formation increases. When the frequency rises above some critical value, the sudden transition to ligament formation from direct drop formation appears, as shown in Fig. 1-b. With further increase of the flow rate, the ligaments become thicker and longer and finally lose their capability of removing all the liquid from the edge, and a thin liquid film is formed around the disk, as shown in Fig. 1-c.

The transition between these phenomena of liquid disintegration by a spinning disk has been studied by a few investigators ${ }^{1,2,6)}$. Tanasawa et al. ${ }^{6)}$ gave an experimental equation for giving the transition condition from direct drop formation to ligament formation. The transition flow rate $Q_{1}$ was given by

\footnotetext{
* Received on July 20, 1973

Presented at the 7th Autumn Meeting of The Soc. of Chem. Engrs., Japan (Nagoya, Oct. 1973)

₹152 東京都目黒区大岡山2-12-1

東京工業大学原子炉工学研究所 松本史朗
}

$$
Q_{1}=2.8\left(\frac{D}{N}\right)^{2 / 3}\left(\frac{\sigma}{\rho}\right) /\left\{1+10\left(\frac{\mu}{\sqrt{\rho \sigma D}}\right)^{1 / 3}\right\}
$$

where $D$ is the diame er of disk, $N$ the rotational number and $\rho, \mu$ and $\sigma$ the density, the viscosity and the surface tension of liquid, respectively. Concerning the transition from ligament to film formation, Hinze and Milborn $^{2)}$ gave a semi-empirical equation on assuming that film formation would take place when the feed rate of liquid exceeds a certain flow rate within which the liquid can be removed through ligaments.

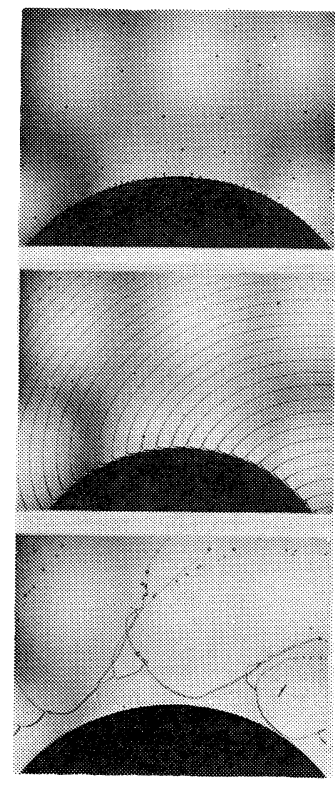

Fig. 1-a

Direct drop formation

$R=6 \mathrm{~cm}$

$N=1000 \mathrm{rpm}$

$\mu \rightleftharpoons 0.598$ poise

$\rho=1.21 \mathrm{~g} / \mathrm{cm}^{3}$

$\sigma=63.0$ dyne $/ \mathrm{cm}$

$Q=2.0 \mathrm{~cm}^{3} / \mathrm{sec}$

Fig. 1-b

Ligament formation

$R=5 \mathrm{~cm}$

$N=1000 \mathrm{rpm}$

$\mu=1.70$ poise

$\rho=1.23 \mathrm{~g} / \mathrm{cm}^{3}$

$\sigma=62.0$ dyne $/ \mathrm{cm}$

$Q=8.0 \mathrm{~cm}^{3} / \mathrm{sec}$

Fig. 1-c

Film formation

$R=6 \mathrm{~cm}$

$N=1000 \mathrm{rpm}$

$\mu=0.598$ poise

$\rho=1.21 \mathrm{~g} / \mathrm{cm}^{3}$

$\sigma=63.0$ dyne $/ \mathrm{cm}$

$Q=16.8 \mathrm{~cm}^{3} / \mathrm{sec}$

Fig. 1 Photographs of three different types of atomization 

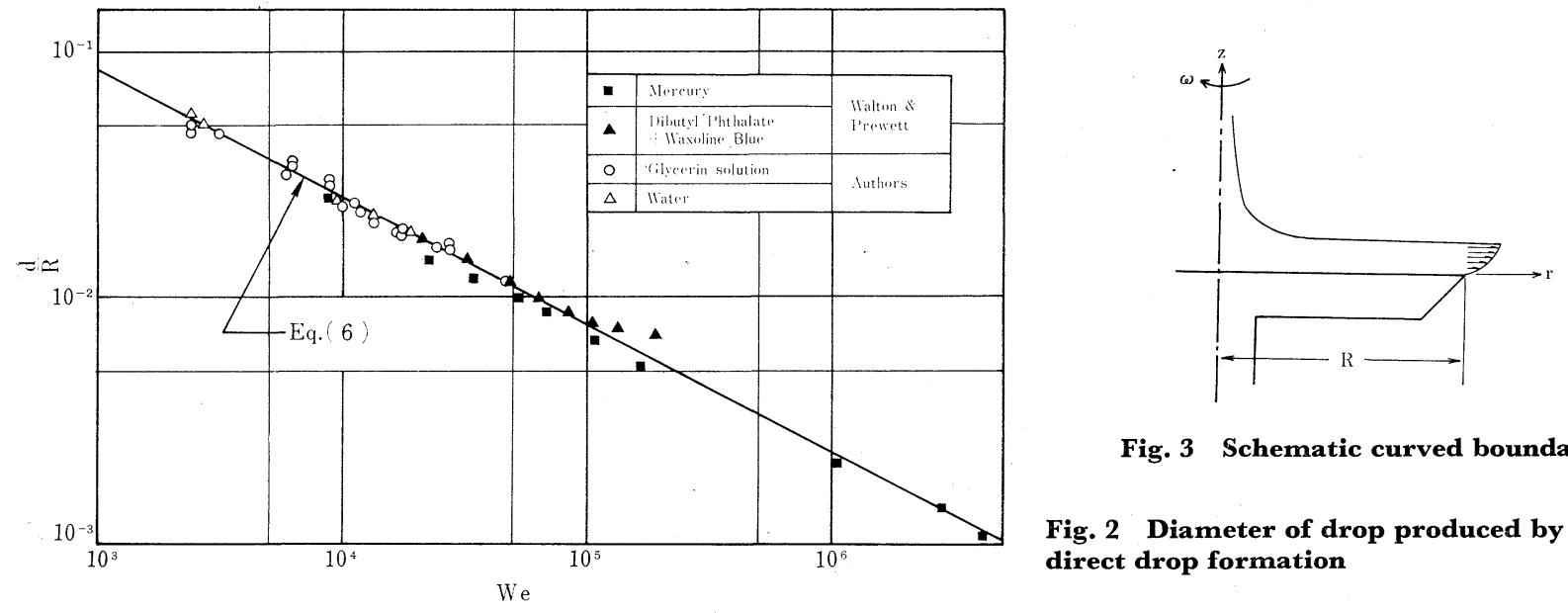

Fig. 3 Schematic curved boundary

Fig. 2 Diameter of drop produced by direct drop formation

This flow rate $Q_{2}$ was given by

$$
\frac{\rho Q_{2}^{2}}{\sigma R^{3}}\left(\frac{\rho R^{3} \omega^{2}}{\sigma}\right)^{0.6}\left(\frac{\mu^{2}}{\rho \sigma R}\right)^{1 / 6}=4.56
$$

where $R$ is the radius of the disk and $\omega$ the angular speed of the disk.

Tanasawa et al. $\left.{ }^{6}\right)$ also gave semi-empirical equations. For viscous liquids $(D \rho / \mu<30)$, the transition flow rate $Q_{2}$ was given by

$$
Q_{2}=5.3\left(\frac{D}{N}\right)^{2 / 3}\left(\frac{\sigma}{\rho}\right)\left(\frac{\rho}{\mu}\right)^{1 / 3}
$$

Fraser et al. ${ }^{1)}$ assumed that the transition occurs when the contracting velocity of the film is larger than the radial velocity of liquid leaving the disk, and gave a semi-empirical equation as follows:

$$
\left(\frac{\rho R^{3} \omega^{2}}{\sigma}\right)\left(\frac{\bar{Q}_{2}}{R^{3} \omega}\right)\left(\frac{R \mu}{\rho \bar{Q}_{2}}\right)^{0.19}=0.636
$$

where $\overline{Q_{2}}$ is the transition flow rate from film to ligament formation.

In this paper, three dimensionless parameters relating to the two transitions of liquid disintegration have been derived theoretically from two simple models. Their transition flow rates have been correlated in terms of these dimensionless parameters experimentally. The experimental results have been compared with the above mentioned semi-empirical equations which are expressed by the three dimensionless parameters.

\section{Theoretical Consideration}

Firstly, we consider the transition from direct drop to ligament formation. Denoting the number of drops appearing on the disk edge $n$, the diameter of drop removed from the disk $d$, and the leaving frequency of a drop from the disk $\tau$, the number of drops removed per second becomes $n \tau$ and from the mass balance,

$$
\tau^{+}=\frac{\tau}{\omega}=\frac{6 Q_{1}}{\pi n d^{3} \omega}
$$

where $\tau^{+}$is the dimensionless frequency, $Q_{1}$ the flow rate and $\omega$ the angular velocity. It is assumed that the transition from direct drop to ligament formation occurs when $\tau^{+}$is larger than some constant.

The diameter of drops produced by direct drop formation was obtained by Walton and Prewett ${ }^{\text {?) }}$.

$$
\frac{d}{R}=2.69 W e^{-1 / 2}
$$

This equation was confirmed by the authors' experimental results as shown in Fig. 2.

The number of local positions in which the drop grows along the disk edge will be same as that of ligaments when the transition occurs. The number of ligaments was studied by Hinze and Milborn'2), and $\mathrm{Kamiya}^{3)}$. The semi-empirical equation of Hinze and Milborn is given by

$$
n=b W e^{1 / 4} R e^{1 / 3}
$$

where $W e$ and $R e$ are defined by

$$
W e=\frac{\rho R^{3} \omega^{2}}{\sigma}, \quad R e=\frac{R^{2} \omega}{\nu}
$$

From Eqs.(5), (6) and (7), the dimensionless transition flow rate $Q_{1}^{+}$is given by

$$
Q_{1}^{+}=C R e^{5 / 6} W e^{-5 / 4}
$$

where $Q_{1}^{+}$is defined by

$$
Q_{1}^{+}=\frac{Q_{1}}{2 \pi R^{2} \sqrt{\nu \omega}}
$$

and $\nu$ is the kinematic viscosity of liquid.

However, the constant $C$ must be determined experimentally.

Next, we consider the transition from film to ligament formation by assuming that the transition occurs when the surface force is overcome by the force of inertia and the centrifugal force at the free boundary beyond the edge of a rotating disk. Further, it is assumed that the free boundary forms a curved boundary corresponding to the radial velocity distribution at the edge of the disk, as shown in Fig. 3.

The force of inertia of liquid removed from the disk is given by

$$
F_{I}=\int_{0}^{2 \pi} \int_{0}^{z_{0}} \rho u^{2} R d \theta d z
$$


where $u$ is the radial velocity of liquid and $z_{0}$ the surface position from the disk (film thickness).

The centrifugal force of liquid removed from the disk is given by

$$
F_{C}=\int_{0}^{2 \pi} \int_{0}^{z_{0}} \rho \frac{v^{2}}{R} \frac{u}{\omega} R d \theta d z
$$

where $v$ is the tangential velocity of liquid.

If the curved boundary of liquid removed from the disk is approximated by

$$
r=R+\frac{u}{\omega}=R(1+F)
$$

Eq.(13) presents one radius of the curvature $R_{1}$. The other radius $R_{2}$ is obtained by

$$
R_{2}=\left\{1+\left(\frac{d z}{d r}\right)^{2}\right\}^{3 / 2} /\left|\frac{d^{2} r}{d z^{2}}\right|
$$

Also, the surface element, $d s$, on the curved boundary is obtained from

$$
d s=2 \pi r \sqrt{1+\left(\frac{d r}{d z}\right)^{2}}
$$

Therefore, the surface force at the free boundary of liquid removed from the disk is given by

$$
F_{S}=\int_{0}^{z_{0}} \sigma\left(\frac{1}{R_{1}}+\frac{1}{R_{2}}\right) d s d z
$$

Previous dimensionless flow rate $Q^{+}$is given by ${ }^{4,5)}$

$$
Q^{+}=\frac{Q}{2 \pi R^{2} \sqrt{\nu \omega}}=\int_{0}^{\hat{s}_{0}} F d \xi
$$

where $F$ is the dimensionless radial velocity divided by the rotating speed of the disk, $R \omega$, and $\xi$ the dimensionless distance from the disk defined by

$$
\xi=z \sqrt{\frac{\omega}{\nu}}
$$

Thus, $\xi_{0}$ denotes the dimensionless film thickness on the disk.

By using the rotating speed and Eq.(18), Eqs.(11), (12) and (16) are transformed into the following equations.

$$
\begin{gathered}
F_{I}=2 \pi \rho R^{3} \omega \sqrt{\nu \omega} \int_{0}^{\xi_{0}} F^{2} d \xi \\
F_{C}=2 \pi \rho R^{3} \omega \sqrt{\nu \omega} \int_{0}^{\xi_{0}} F G^{2} d \xi \\
F_{S}=2 \pi \sigma \sqrt{\frac{\nu}{\omega}} \int_{0}^{\hat{\xi}_{0}} \frac{\left\{1+\frac{R^{2} \omega}{\nu}\left(F^{\prime}\right)^{2}\right\}^{3 / 2}+\frac{R^{2} \omega}{\nu}\left|F^{\prime \prime}\right| F}{1+\frac{R^{2} \omega}{\nu}\left(F^{\prime}\right)^{2}} d \xi
\end{gathered}
$$

where $G$ is the dimensionless tangential velocity.

Thus the ratio, $C^{\prime}$ of both the force of inertia and the centrifugal force to the surface force is given by

$$
C^{\prime}=\frac{W e \int_{0}^{\hat{\xi}_{0}}\left(F^{2}+F G^{2}\right) d \xi}{\int_{0}^{\xi_{0}} \frac{\left\{1+\operatorname{Re}\left(F^{\prime}\right)^{2}\right\}^{3 / 2}+R e\left|F^{\prime \prime}\right| F}{1+\operatorname{Re}\left(F^{\prime}\right)^{2}} d \xi}
$$

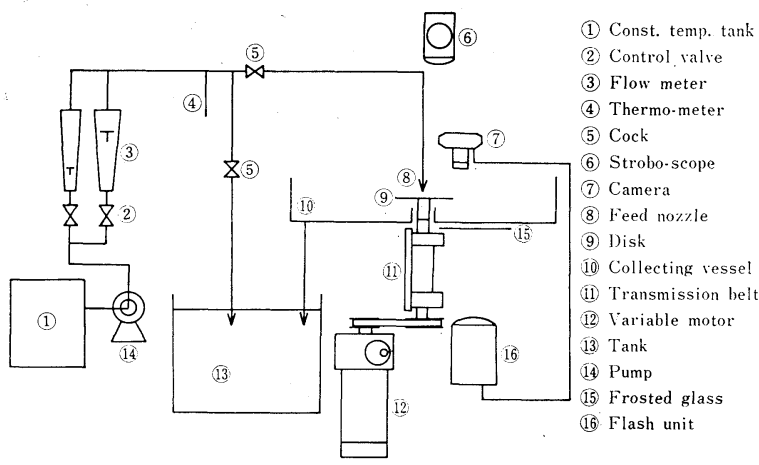

Fig. 4 Schematic diagram of experimental apparatus

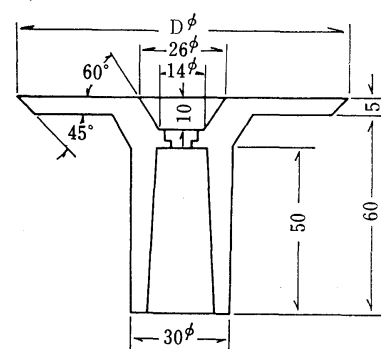

Fig. 5 Detail of disk

Therefore, it is assumed that if the ratio, $C^{\prime}$, is smaller than some constant, the transition will take place from film to ligament formation, and if the ratio is larger than the constant, the reverse will occur. However, $C^{\prime}$ must be determined experimentally by calculating Eq.(22) under many experimental data. In this paper, we consider the functional correlation among the three dimensionless parameters, $R e, W e$ and $\xi_{0}$. The numerator in Eq.(22) is a function of $W e$ and $\xi_{0}$, the denominator a function of $R e$ and $\xi_{0}$. From Eq. (17), $\xi_{0}$ can be determined from $Q^{+}$.

Therefore, $C^{\prime}$ can be given by the following functional correlation.

$$
C^{\prime}=f\left(R e, W e, Q^{+}\right)
$$

Thus the transition flow rate from film to ligament formation and the reverse may be correlated experimentally in terms of $R e$ and $W e$.

Eqs. (2), (3) and (4) can be rearranged by these parameters as follows:

Hinze and Milborn:

$$
Q_{2}^{+}=0.340 \frac{R e^{2 / 3}}{W e^{0.883}}
$$

Tanasawa et al.:

$$
Q_{2}^{++}=0.297 \frac{R e^{6 / 5}}{W e}
$$

Fraser et al.:

$$
\bar{Q}_{2}^{+}=0.108 \frac{R e^{2 / 3}}{W e^{0.875}}
$$

\section{Experimental}

A flow diagram of the experimental apparatus is shown in Fig. 4. The detail of disks is shown in Fig. 5. Both the flow rate and the rotational speed have been 


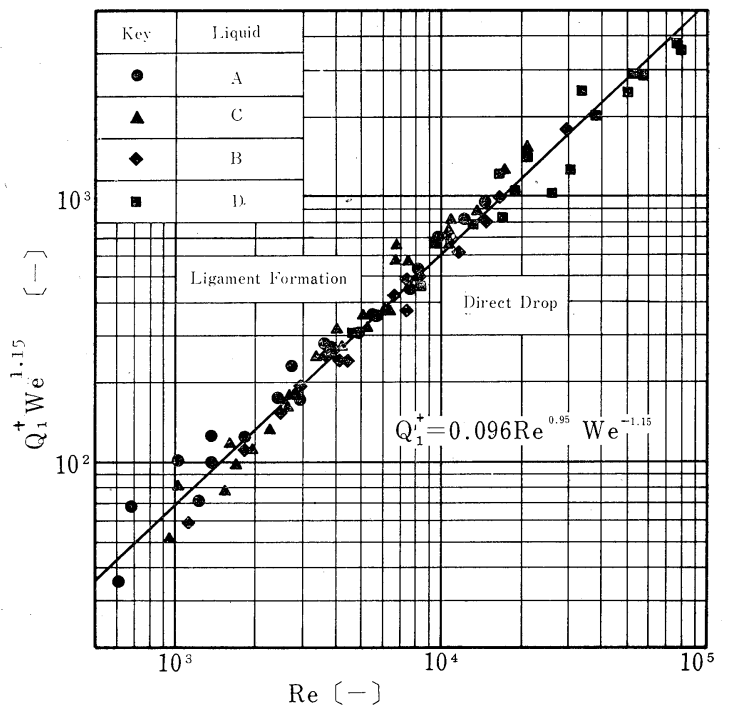

Fig. 6 Transition from direct drop to ligament formation

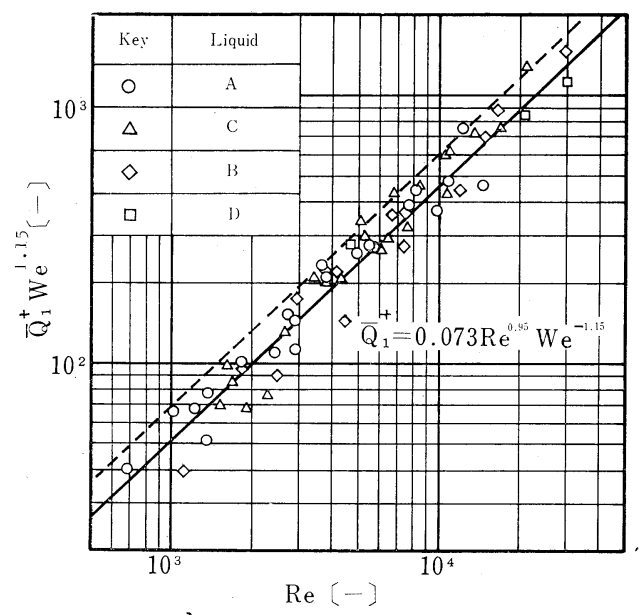

Fig. 7 Transition from ligament to direct drop formation

varied to such an extent that three different types of disintegration have been achieved. The transition has been observed directly with a stroboscope.

The physical properties of liquid used in this experiment are given in Table 1. The density, the viscosity and the surface tension of liquid were measured by an ordinary hydrometer, a viscometer of Ubellode type and a Denouy tensiometer, respectively.

\section{Result and Discussion}

The representative results obtained in this experiment are listed in Table 2, where $\bar{Q}_{1}$ and $\overline{Q_{2}}$ are the transition flow rate from ligament to direct drop formation and from film to ligament formation, respectively.

The correlation expressed by Eq.(9) for the transition from direct drop to ligament formation gives good agreement with the experimental results. However, a little better correlation given by Eq.(27) has been obtained, as shown in Fig. 6.

In the case of the reverse process, the condition of transition happens at a slightly smaller flow rate, as

\begin{tabular}{|c|c|c|c|c|}
\hline \multicolumn{2}{|r|}{ Table 1} & \multicolumn{3}{|c|}{ Physical properties of liquid } \\
\hline \multicolumn{2}{|c|}{ Liquid } & Density & Viscosity & $\begin{array}{l}\text { Surface } \\
\text { tension }\end{array}$ \\
\hline$A$ & $\begin{array}{l}\text { Glycerine } \\
\text { solution }\end{array}$ & 1.23 & 1.70 & 62.0 \\
\hline$B$ & $\begin{array}{l}\text { Ethanol + } \\
\text { Glycerine } \\
\text { solution }\end{array}$ & 1.09 & 0.495 & 31.0 \\
\hline C & $\begin{array}{l}\text { Glycerine } \\
\text { solution }\end{array}$ & 1.21 & 0.598 & 63.0 \\
\hline$D$ & $\begin{array}{l}\text { Glycerine } \\
\text { solution }\end{array}$ & 1.16 & 0.115 & 64.2 \\
\hline
\end{tabular}

Table 2 Experimental results of transition flow rate

\begin{tabular}{|c|c|c|c|c|c|c|}
\hline Liquid & $\begin{array}{c}N \\
{[\mathrm{rpm}]}\end{array}$ & $\begin{array}{c}R \\
{[\mathrm{~cm}]}\end{array}$ & $\begin{array}{c}Q_{1} \\
{\left[\mathrm{~cm}^{3} /\right.} \\
\mathrm{sec}]\end{array}$ & $\begin{array}{l}\bar{Q}_{1} \\
{\left[\mathrm{~cm}^{3} /\right.} \\
\mathrm{sec}]\end{array}$ & $\begin{array}{c}Q_{2} \\
{\left[\mathrm{~cm}^{3} /\right.} \\
\mathrm{sec}]\end{array}$ & $\begin{array}{c}\bar{Q}_{2} \\
{\left[\mathrm{~cm}^{3} /\right.} \\
\mathrm{sec}]\end{array}$ \\
\hline \multirow{9}{*}{$A$} & 1000 & 3 & 2.15 & 1.25 & 9.65 & 7.25 \\
\hline & 2000 & 3 & 0.90 & 0.90 & 6.00 & 4.85 \\
\hline & 2000 & 4 & 1.05 & 0.65 & 7.06 & 5.88 \\
\hline & 3000 & 5 & 0.75 & 0.70 & 6.26 & 5.07 \\
\hline & 2000 & 6 & 1.20 & 0.90 & 8.84 & 7.90 \\
\hline & 3000 & 6 & 0.85 & 0.70 & 6.90 & 6.00 \\
\hline & 3000 & 8 & 1.00 & 0.48 & 7.87 & 7.65 \\
\hline & 4000 & 5 & 0.56 & 0.48 & 4.88 & 4.58 \\
\hline & 4000 & 6 & 0.65 & 0.45 & 5.84 & 5.17 \\
\hline \multirow{11}{*}{$C$} & 300 & 5 & 9.53 & 8.00 & 34.2 & 28.1 \\
\hline & 500 & 3 & 3.48 & 2.00 & 17.1 & 15.5 \\
\hline & 500 & 5 & 5.18 & 4.20 & 23.8 & 19.2 \\
\hline & 500 & 6 & 6.40 & 5.10 & 26.2 & 20.1 \\
\hline & 500 & 10 & 5.10 & 4.36 & 22.1 & 20.1 \\
\hline & 1000 & 3 & 2.15 & 1.30 & 11.4 & 8.19 \\
\hline & 1000 & 5 & 3.01 & 2.70 & 16.1 & 12.7 \\
\hline & 1000 & 8 & 4.10 & 3.40 & 21.1 & 17.5 \\
\hline & 2000 & 4 & 2.13 & 1.60 & 9.25 & 8.00 \\
\hline & 2000 & 5 & 2.00 & 1.60 & 10.9 & 10.0 \\
\hline & 500 & 3 & 10.2 & 9.00 & 22.4 & 21.2 \\
\hline \multirow{6}{*}{$D$} & 800 & 5 & 9.50 & 5.70 & 25.7 & 19.6 \\
\hline & 800 & 6 & 6.40 & 5.80 & 23.7 & - \\
\hline & 1000 & 3 & 6.40 & - & 15.3 & 12.5 \\
\hline & 1000 & 6 & 7.00 & - & 18.0 & 14.0 \\
\hline & 2000 & 3 & - & - & 10.4 & 8.55 \\
\hline & 2000 & 6 & 3.70 & - & 11.9 & - \\
\hline \multirow{6}{*}{$B$} & 1000 & 4 & 1.55 & 1.34 & 8.26 & 6.53 \\
\hline & 2000 & 4 & 0.86 & 0.65 & 4.80 & 4.40 \\
\hline & 1000 & 6 & 1.70 & 1.55 & 9.23 & 8.80 \\
\hline & 2000 & 6 & 0.96 & 0.86 & 6.95 & 5.79 \\
\hline & 1000 & 8 & 1.80 & 1.58 & 12.2 & 11.7 \\
\hline & 2000 & 8 & 1.14 & 0.94 & 8.13 & 7.30 \\
\hline
\end{tabular}

shown in Fig. 7. The dash line in Fig. 7 represents the best fit line in Fig. 6 .

Therefore, the recommendable experimental equations are

$$
\begin{aligned}
& Q_{1}^{+}=0.096 \frac{R e^{0.95}}{W e^{1.15}} \\
& \bar{Q}_{1}^{+}=0.073 \frac{R e^{0.95}}{W e^{1.15}}
\end{aligned}
$$

Figure 8 shows the transition from ligament to film formation. The reverse transition is shown in Fig. 9. In these figures, the two-point dash line represents Eq.(24) and the dash line Eq.(26).

Hinze and Milborn's equation for the transition from ligament to film formation gives fairly good a- 


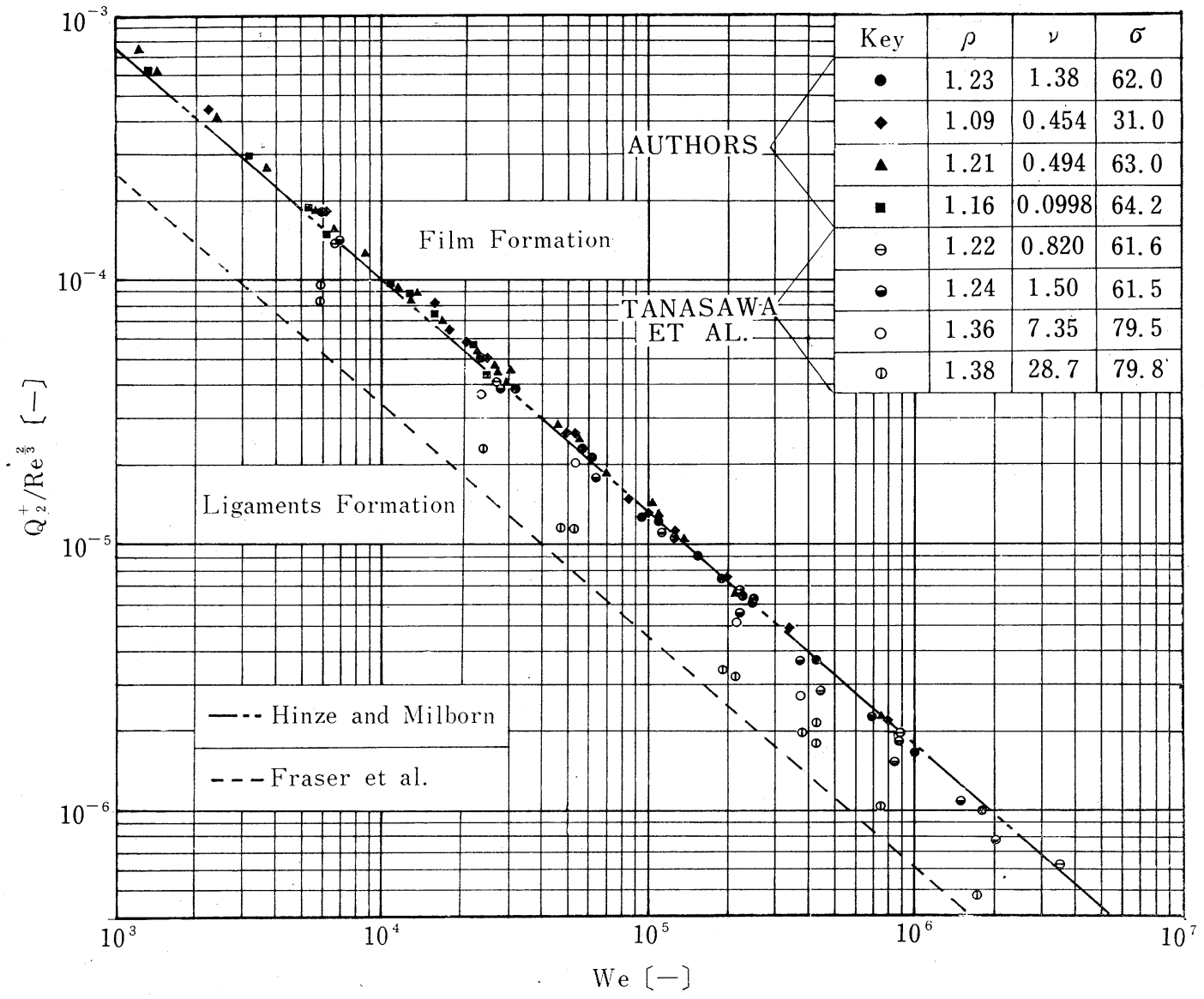

Fig. 8 Transition from ligament formation to film formation

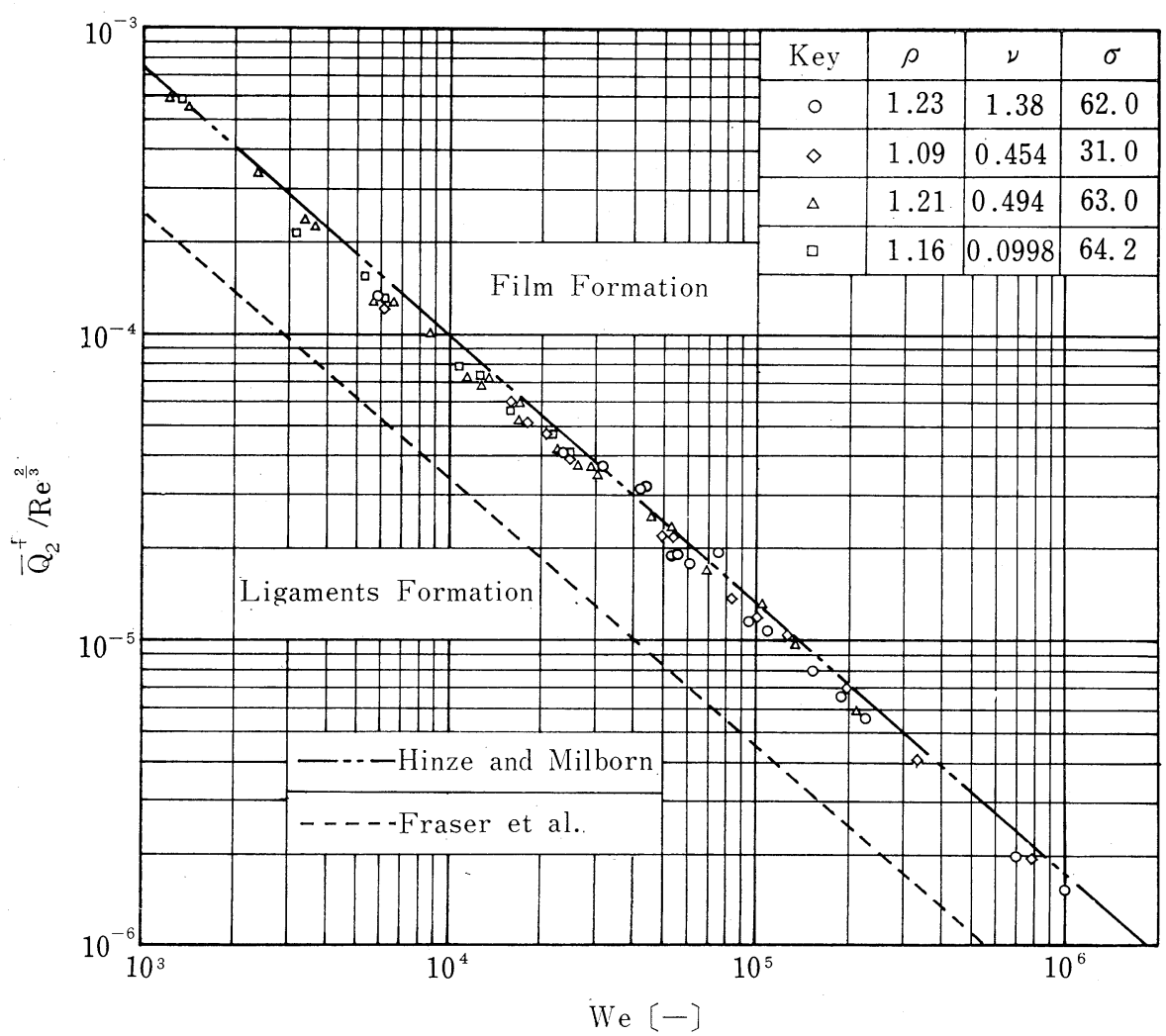

Fig. 9 Transition from film formation to ligament formation

greement with the authors' experimental results, as shown in Figs. 8 and 9. On the other hand, the semiempirical equation by Fraser et al. gives lower values than the experimental results.
A comparison between the semi-empirical equation by Tanasawa et al. and the experimental results is shown in Figs. 10 and 11. In these figures, solid line represents Eq.(25). For the transition from film to 


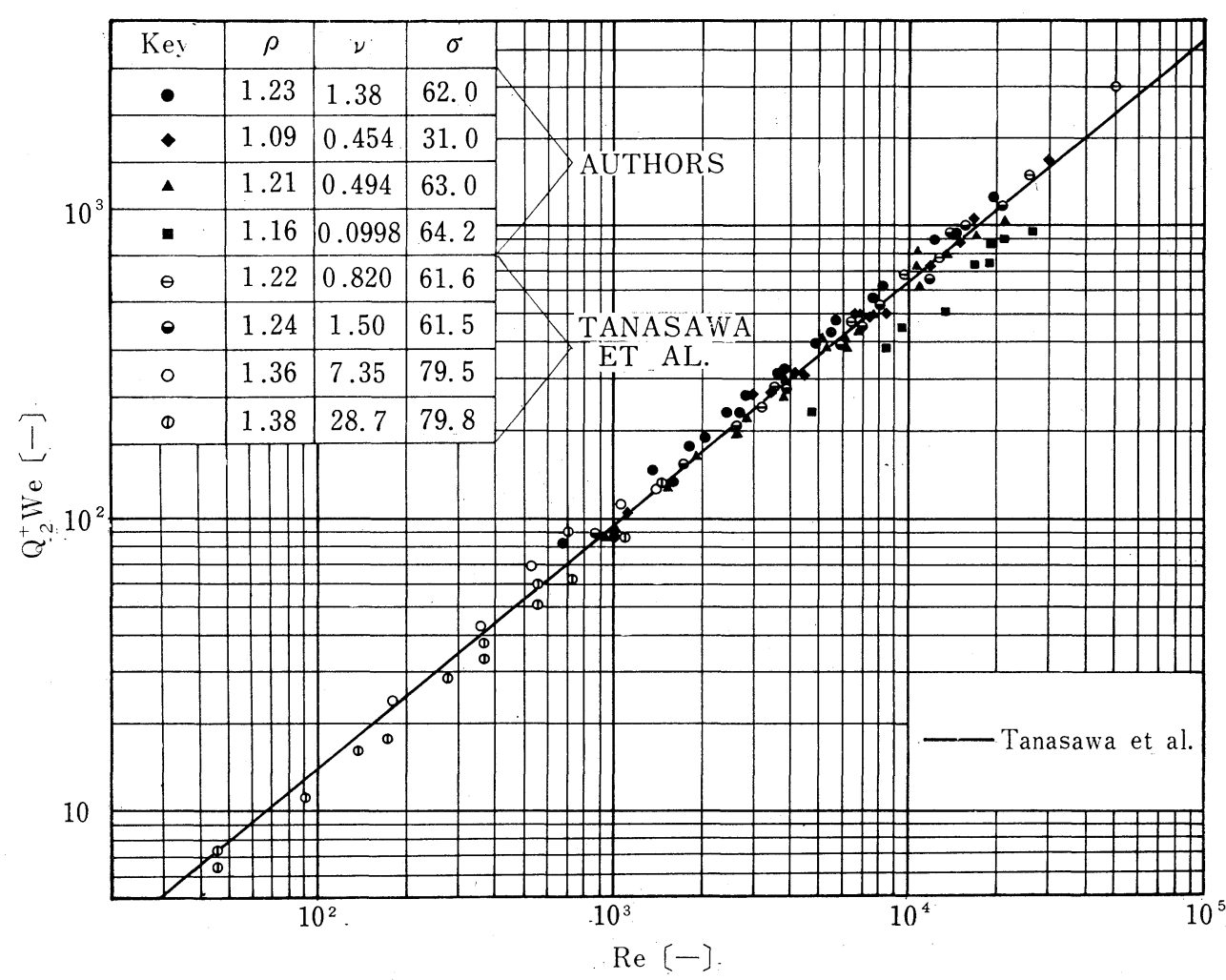

Fig. 10 Transition from ligament formation to film formation

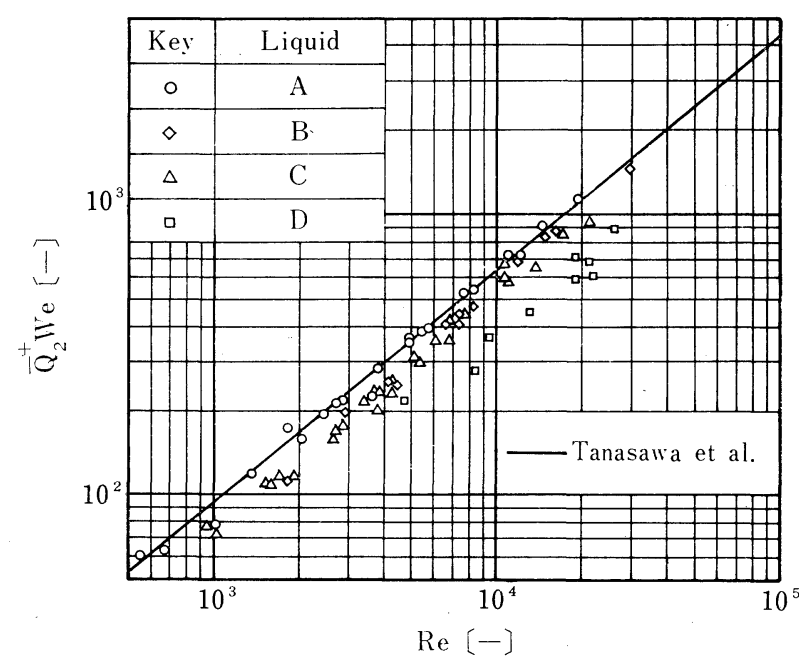

Fig. 11 Transition from film formation to ligament formation

ligament formation, the effect of the viscosity of liquid was not sufficiently taken into consideration in Eq.(25), as shown in Fig. 11. However, the reverse transition flow rate gives fairly good agreement with the experimental results except for the data of low viscous liquid, as shown in Fig. 10. This equation is originally a semiempirical equation made for the transition flow rate from ligament to film formation using highly viscous liquids.

However, Hinze and Milborn's equation does not fit for very viscous liquid, though it does very well for liquid of less than a few poises in viscosity.

Previous three semi-empirical equations for the transition fow rate from ligament to film formation were compared with many experimental results. It was seen that none of the equations could give good correlation among $R e, W e$ and $Q^{+}$over a wide range of operating conditions.

\section{Conclusion}

The transition flow rate of three different types of disintegration were studied. Eqs. (27) and (28) were obtained from the experimental results at the transition from direct drop to ligament formation and at the reverse transition, respectively. On the other hand, three semi-empirical equations of transition flow rate from ligament to film formation or the reverse were rearranged by three dimensionless parameters, $R e, W e$ and $Q^{+}$and compared with various experimental results. For the transition flow rate from ligament to film formation or the reverse, Hinze and Milborn's equation can be used for liquid of less than a few poises in viscosity. For more viscouss liquid, the equation by Tanasawa et al. is applicable for prediction of the transition flow rate.

\section{Acknowledgment}

One of the authors (S. Matsumoto) was granted a scholarship from the Sakkokai Foundation, and expresses his appreciation for the support.

\section{Nomenclature}

$=$ diameter of drop

$=$ dimensionless radial velocity $(u / R \omega)$

$=$ force of inertia

$=$ centrifugal force 


\begin{tabular}{|c|c|c|c|}
\hline$F_{S}$ & $=$ & surface force & [dyne] \\
\hline$G$ & $=$ & dimensionless tangential velocity $(v / R \omega)$ & {$[-]$} \\
\hline$N$ & $=$ & rotational number & {$[-]$} \\
\hline$n$ & $=$ & number of ligaments & {$[-]$} \\
\hline$Q_{1}$ & $=$ & $\begin{array}{l}\text { transition flow rate from direct drop } \\
\text { formation to ligament formation }\end{array}$ & {$\left[\mathrm{cm}^{3} / \mathrm{sec}\right]$} \\
\hline $\bar{Q}_{1}$ & $=$ & $\begin{array}{l}\text { transition flow rate from ligament formation } \\
\text { to direct drop formation }\end{array}$ & {$\left[\mathrm{cm}^{3} / \mathrm{sec}\right]$} \\
\hline$Q_{2}$ & $=$ & $\begin{array}{l}\text { transition flow rate from ligament formation } \\
\text { to film formation }\end{array}$ & {$\left[\mathrm{cm}^{3} / \mathrm{secl}\right.$} \\
\hline $\bar{Q}_{2}$ & $=$ & $\begin{array}{l}\text { transition flow rate from film formation } \\
\text { to ligament formation }\end{array}$ & {$\left[\mathrm{cm}^{3} / \mathrm{sec}\right]$} \\
\hline$R$ & $=$ & radius of disk & {$[\mathrm{cm}]$} \\
\hline$R_{1}$ & $=$ & principal radius of curvature & {$[\mathrm{cm}]$} \\
\hline$R_{2}$ & $=$ & principal radius of curvature & {$[\mathrm{cm}]$} \\
\hline$r$ & $=$ & radial coordinate & {$[-]$} \\
\hline$R e$ & $=$ & Reynolds number $\left(R^{2} \omega / \nu\right)$ & {$[-]$} \\
\hline$s$ & $=$ & surface area & {$\left[\mathrm{cm}^{2}\right]$} \\
\hline$u$ & $=$ & radial velocity & {$[\mathrm{cm} / \mathrm{sec}]$} \\
\hline$v$ & $=$ & tangential velocity & {$[\mathrm{cm} / \mathrm{sec}]$} \\
\hline$W e$ & $=$ & Weber number $\left(\rho R^{3} \omega^{2} / \sigma\right)$ & {$[-]$} \\
\hline$z$ & $=$ & axial coordinate & {$[-]$} \\
\hline$z_{0}$ & $=$ & thickness of liquid film & {$[\mathrm{cm}]$} \\
\hline
\end{tabular}
$\xi \quad=$ dimensionless coordinate
$\xi_{0} \quad=$ dimensionless film thickness
$=$ dimensionless fim thic
$=$ viscosity of liquid
$=$ kinematic viscosity of liquid
$=$ density of liquid
$=$ surface tension of liquid
$=$ leaving frequency of a drop from disk
$=$ dimensionless leaving frequency
$=$ angular speed of disk

$$
\begin{array}{r}
{[-]} \\
{[-]} \\
{[-]} \\
{[\mathrm{g} / \mathrm{cm} \cdot \mathrm{sec}]} \\
{\left[\mathrm{cm}^{2} / \mathrm{sec}^{3}\right]} \\
{\left[\mathrm{g} / \mathrm{cm}^{3}\right]} \\
{\left[\mathrm{dyne} / \mathrm{cm}^{2}\right]} \\
{[1 / \mathrm{sec}]} \\
{[-]} \\
{[\mathrm{radian} / \mathrm{sec}]}
\end{array}
$$

\section{Literature Gited}

1) Fraser, R. P., N. Dombrowski and J. H. Routley: Chem. Eng. Sci., 18, 323 (1963)

2) Hinze, J. O. and H. Milborn: J. Appl. Mech., 17, 145 (1950)

3) Kamiya, T.: J. Chem. Eng. Japan, 5, 391 (1972)

4) Matsumoto, S., K. Saito and Y. Takashima: Bull. Tokyo Inst. Tech., No. 109, 69 (1972), No. 116, 86 (1973)

5) Matsumoto, S., K. Saito and Y. Takashima: J. Chem. Eng. Japan, 6, 503 (1973)

6) Tanasawa, Y., Y. Miyasaka and M. Umehara: Trans. Soc. Mech. Eng. Japan, 25, 879, 888, 897 (1959)

7) Walton, W. H. and W. C. Prewett: Proc. Phys. Soc., 62, 341 (1949)

\title{
SOLID-LIQUID MASS TRANSFER IN FALLING LIQUID FILMS ON SINGLE SPHERES*
}

\author{
Tsutonu HIROSE, Yasunori MORI** and Yuji SATO \\ Department of Chemical Engineering, Kyushu University, Fukuoka
}

\begin{abstract}
Mass transfer equations are derived and compared with dissolution experiments of $\mathrm{C}_{6} \mathrm{H}_{5} \mathrm{COOH}-\mathrm{H}_{2} \mathrm{O}$ and $\mathrm{Cu}-\mathrm{H}_{2} \mathrm{SO}_{4}-\mathrm{K}_{2} \mathrm{Cr}_{2} \mathrm{O}_{7}$ systems on single spheres (6 different sizes from $3 / 4^{\prime \prime}$ to $\left.3^{\prime \prime}\right)$. In region $\mathrm{I}$ (long contact time with smooth laminar film), Sh converges $S h=1.608\left(\operatorname{GaSc} / \mathrm{Pe}^{*}\right)^{1 / 3}$ or $k=2.01 \mathrm{D} / \bar{\delta}$

( $\bar{\delta}$ : mean film thickness) and increases with decreasing volumetric flow rate $Q$. In region II (short contact time with smooth laminar film), Sh converges $S h=0.716 P e^{* 1 / 9}(G a S c)^{2 / 9}$

The transition from region I to II occurs around $P e^{*} /(G a S c)^{1 / 4} \simeq 10$. In region III (short contact time with wavy film surface), $S h$ increases with $P e^{*}$ to the $1 / 3-1 / 2$ power and is substantially higher than that in region II. A generalized short contact time equation using the measured local film thickness $\delta$

$$
S h=0.501 P e^{* 1 / 3}\left\{\int_{0}^{\pi}(d / \delta) \sin \theta d \theta\right\}^{2 / 3}
$$

is valid in this region. Thus, main cause for enhancement in $S h$ is the reduction in film thickness relevant to the wave formation. The II-III transition is somewhere between wave inception and wave coverage flow rates. In the above, $S h=k d / D, P e^{*}=Q / d D$ and $G a=g d^{3} / \nu^{2}$, in which $k$ is based on a log-mean driving force.
\end{abstract}

\section{Introduction}

Gas-liquid mass transfer in spherical falling film has been extensively investigated both theoretically and experimentally ${ }^{3,4,8,14,23)}$ and the results have been used to interpret gas absorption rate in packed column absorbers. It is also recognized that there are many industrial practices where solid-liquid mass transfer in

\footnotetext{
* Received on August 23, 1973

Presented at the 37th Annual Meeting of The Soc. of Chem. Engrs., Japan, C-116, at Nagoya, April 3, 1972

** Toyo Engineering Corp.

于812 福岡市東区箱崎

九州大学工学部化学機械工学科 広瀬 勉
}

falling film on particles is equally important. For example, Satterfield, Pelossof and Sherwood ${ }^{20}$ discussed mass transfer limitation in a trickle-bed catalytic reactor in which reacting gas and liquid components must be transfered to the surface of packing catalyst through falling film on it. In a trickling filter, mass transfer of substrate to the biological film adhering to supporting particles is important ${ }^{1)}$.

Only one analytical paper on solid-liquid mass transfer in spherical falling film has appeared, just recently, for diffusivity measurement ${ }^{15)}$, although some $\mathrm{s}^{7,10,11}$, 17,25) have appeared for inclined flat falling film (mainly in relation to heat transfer problem). Falling film on particles is expected to have different character- 